\title{
Preoperative Chemotherapy for Localized Squamous Cell Carcinoma of the Esophagus? We Should Go Back to the Drawing Board!
}

\author{
Jaffer A. Ajani, $\mathrm{MD}^{\mathbf{1}}$ and Stephen G. Swisher, $\mathrm{MD}^{2}$ \\ ${ }^{1}$ Department of GI Medical Oncology, UTMD Anderson Cancer Center, Houston; ${ }^{2}$ Department of Thoracic and \\ Cardiovascular Surgery, UTMD Anderson Cancer Center, Houston
}

Squamous cell carcinoma is rampant in certain parts of the world and it is the most common histologic subtype of esophageal cancer globally. Compared to adenocarcinoma, where obesity, gastroesophageal reflux, and Barrett esophagus seem to play a role, tumorigenesis of squamous cell carcinoma of the esophagus is associated with smoking, alcohol consumption, and poor nutrition (or nutritional deficiencies). ${ }^{1}$ Recent molecular studies have identified a subgroup of tumor initiating cells for squamous cell carcinoma with TGF- $\beta$ and integrin/focal adhesion kinase (FAK) signaling and a cancer stem cell signature has been correlated with a number of self-renewal genes orchestrated by Bmil, Hmaga2, and Sox $2{ }^{2}$ Genome-wide association studies have also identified three susceptibility loci related to tobacco and alcohol. ${ }^{3}$ Further molecular studies are needed to develop better risk stratification and therapeutics for squamous cell carcinoma patients.

For patients with localized squamous cell carcinoma, surgery as primary therapy is commonly used in many regions of the world, but results are uniformly poor. ${ }^{4} \mathrm{We}$ recommend surgery for patients with $\mathrm{T} 1 \mathrm{~b}$ cancer, but patients with tumors higher that $\mathrm{T} 1 \mathrm{~b}$ should be considered for combined modality therapy. Preoperative chemoradiation seems to provide the strongest level 1 evidence based on the Dutch trial of preoperative chemoradiation versus surgery. ${ }^{5}$

In this issue, Ando et al. discuss their results of a phase III trial comparing preoperative cisplatin/fluorouracil with postoperative cisplatin/fluorouracil in patients with

(C) Society of Surgical Oncology 2011

Published Online: 12 October 2011

J. A. Ajani, MD

e-mail: Jajani@mdanderson.org localized squamous cell carcinoma of the esophagus. A total of 330 patients were randomized, and the authors found that the overall survival (OS) of patients who received preoperative chemotherapy was superior to that for patients who received postoperative chemotherapy. The authors claim that preoperative chemotherapy should therefore be regarded as standard treatment.

We acknowledge that the conception and execution of a phase III trial requires mobilization of many resources, including commitment of many dedicated investigators, time, cost, and labor. However, a phase III trial, if properly conceived and completed, provides the best tool to sort out the true contributions of a therapy or approach of interest.

Let us examine the trial being published by Ando et al. The current trial was based on their previous experience with JCOG9204, which randomized 242 patients with squamous cell carcinoma to surgery alone or postoperative cisplatin/fluorouracil. ${ }^{6}$ The primary end point of diseasefree survival (DFS) was increased in the postoperative chemotherapy arm $(P=0.037)$ but the overall survival was not different for the two groups $(P=0.13)$. A subgroup analysis of JCOG9204 showed that patients with node-negative cancer did not benefit from postoperative chemotherapy. The current trial randomized localized squamous cell carcinoma patients to receive either postoperative chemotherapy or preoperative chemotherapy with the primary end point of DFS. Unfortunately, the trial design of this study had a major flaw that precludes any meaningful conclusions to be drawn. In the postoperative treatment arm, patients with node negative cancer did not receive chemotherapy because JCOG 9204 did not find a benefit for adjuvant chemotherapy in a subset analysis of node-negative patients. Because of this design, 140 patients receive preoperative chemotherapy, while only 81 patients 
(with node-positive cancer) received postoperative chemotherapy. Thus, the postoperative treatment arm treated only node-positive patients (apples), whereas the preoperative treatment group treated both node-positive and nodenegative patients (oranges). This imbalance in treatment arms therefore does not allow us to conclude that preoperative therapy is superior to postoperative therapy because not all patients in the postoperative chemotherapy arm received treatment. Additionally, the primary end point of DFS was not met $(P=0.08)$, yet overall survival was in favor of the preoperative group $(P=0.01)$. The authors do not provide cancer-specific mortality information and do not provide information on several patients who did not receive therapy in the postoperative arm. It is also not clear whether unstratified analyses included those patients who were not treated in the postoperative arm. We agree with the authors that surgery alone is inadequate for this group of patients, but we cannot agree that the current study establishes the superiority of preoperative therapy over postoperative therapy.

As the contradictions in this study demonstrate, there may be no single treatment that works for all groups of localized squamous cell carcinoma of the esophagus. Indeed, it may be time to go back to the drawing board: our future hopes may no longer rest in establishing refined empiric approaches, but rather in developing individualized approaches of optimizing therapy to patients on the basis of imaging, molecular biomarkers, and patient genetics.

\section{REFERENCES}

1. Lagergren J. Influence of obesity on the risk of esophageal disorders. Nat Rev Gastroenterol Hepatol. 2011;8:340-7.

2. Schober M, Fuchs E. Tumor-initiating stem cells of squamous cell carcinomas and their control by TGF- $\beta$ and integrin/focal adhesion kinase (FAK) signaling. Proc Natl Acad Sci USA. 2011;108: 10544-9.

3. $\mathrm{Wu} \mathrm{C}, \mathrm{Hu} \mathrm{Z}, \mathrm{He} \mathrm{Z}$, et al. Genome-wide association study identifies three new susceptibility loci for esophageal squamous-cell carcinoma in Chinese populations. Nat Genet. 2011;43:679-84.

4. Rice TW, Rusch VW, Apperson-Hansen C, et al. Worldwide esophageal cancer collaboration. Dis Esophagus. 2009;22:1-8.

5. Gaast AV, van Hagen P, Hulshof M, et al. Effect of preoperative concurrent chemoradiotherapy on survival of patients with resectable esophageal or esophagogastric junction cancer: results from a multicenter randomized phase III study. Proceeding American Society of Clinical Oncology. Chicago, IL: ASCO; 2010.

6. Ando N, Iizuka $\mathrm{T}$, Ide $\mathrm{H}$, et al. Surgery plus chemotherapy compared with surgery alone for localized squamous cell carcinoma of the thoracic esophagus: a Japan Clinical Oncology Group Study JCOG9204. J Clin Oncol. 2003;21:4592-6. 